\title{
ANISOTROPIC SOBOLEV HOMEOMORPHISMS
}

\section{Patrizia Di Gironimo, Luigi D'Onofrio, Carlo Sbordone and Roberta Schiattarella}

Università degli Studi di Salerno, Dipartimento di Matematica e Informatica

Via Ponte don Melillo, 84084 Fisciano (SA), Italy; pdigironimo@unisa.it

Università degli Studi di Napoli "Parthenope", Dipartimento di Statistica e Matematica per la Ricerca Economica, Via Medina 40, 80131 Napoli, Italy; donofrio@uniparthenope.it

Università degli Studi di Napoli Federico II, Dipartimento di Matematica e

Applicazioni "R. Caccioppoli", Via Cintia, 80126 Napoli, Italy; sbordone@unina.it

Università degli Studi di Napoli Federico II, Dipartimento di Matematica e

Applicazioni "R. Caccioppoli", Via Cintia, 80126 Napoli, Italy; roberta.schiattarella@unina.it

Abstract. Let $\Omega \subset \mathbf{R}^{2}$ be a domain. Suppose that $f \in \mathscr{W}_{\text {loc }}^{1,1}\left(\Omega ; \mathbf{R}^{2}\right)$ is a homeomorphism. Then the components $x(w), y(w)$ of the inverse $f^{-1}=(x, y): \Omega^{\prime} \rightarrow \Omega$ have total variations given by

$$
|\nabla y|\left(\Omega^{\prime}\right)=\int_{\Omega}\left|\frac{\partial f}{\partial x}\right| d z, \quad|\nabla x|\left(\Omega^{\prime}\right)=\int_{\Omega}\left|\frac{\partial f}{\partial y}\right| d z .
$$

\section{Introduction}

Let $\Omega \subseteq \mathbf{R}^{2}$ and $\Omega^{\prime} \subseteq \mathbf{R}^{2}$ be domains. Recently, homeomorphisms $f=(u, v): \Omega$ $\stackrel{\text { onto }}{\longrightarrow} \Omega^{\prime}$ which are a.e. differentiable together with their inverses $f^{-1}=(x, y): \Omega^{\prime} \stackrel{\text { onto }}{\longrightarrow}$ $\Omega$ have been intensively studied (see [9], [11]).

A homeomorphism $f: \Omega \stackrel{\text { onto }}{\longrightarrow} \Omega^{\prime}$ which belongs to the Sobolev space $\mathscr{W}_{\text {loc }}^{1,1}\left(\Omega ; \mathbf{R}^{2}\right)$ is called a $\mathscr{W}^{1,1}$-homeomorphism. If also $f^{-1}$ is a $\mathscr{W}^{1,1}$-homeomorphism, we say that $f$ is a bi-Sobolev map (see [13]). We recall that a $\mathscr{W}^{1,1}$-homeomorphism is differentiable a.e. thanks to the well known Gehring-Lehto Theorem (see [6], Theorem 2).

If we adopt the following notations:

$$
\begin{aligned}
& f(x, y)=(u(x, y), v(x, y)) \quad \text { for }(x, y) \in \Omega, \\
& f^{-1}(u, v)=(x(u, v), y(u, v)) \quad \text { for }(u, v) \in \Omega^{\prime},
\end{aligned}
$$

then the bi-Sobolev condition for $f$ and $f^{-1}$ can be precisely expressed by

$$
u_{x}, u_{y}, v_{x}, v_{y} \in L_{\mathrm{loc}}^{1}(\Omega)
$$

and

$$
x_{u}, x_{v}, y_{u}, y_{v} \in L_{\mathrm{loc}}^{1}\left(\Omega^{\prime}\right) .
$$

The following result derives from [3],[9] and [13].

Theorem 1.1. If $f: \Omega \stackrel{\text { onto }}{\longrightarrow} \Omega^{\prime}$ is a bi-Sobolev map, then

$$
\int_{\Omega}|D f| d z=\int_{\Omega^{\prime}}\left|D f^{-1}\right| d w .
$$

doi:10.5186/aasfm.2011.3632

2010 Mathematics Subject Classification: Primary 46E35, 26B30.

Key words: Sobolev homeomorphisms.

This paper was performed while the third author was a Professor at the "Centro Interdisciplinare Linceo Beniamino Segre" of the Accademia Nazionale dei Lincei, Rome. 
If $f$ is an a.e. differentiable homeomorphism, then the Jacobian determinant $J_{f}$ satisfies either the inequality $J_{f} \geq 0$ or $J_{f} \leq 0$ a.e. ([2], [12]). For simplicity let us assume $J_{f}(z) \geq 0$ for a.e. $z \in \Omega$.

Let us point out that if the Jacobians $J_{f}$ of $f$ and $J_{f^{-1}}$ of $f^{-1}$ are strictly positive a.e., it is possible to prove (1.3) by mean of the area formula (see Sections 2 and 3). On the other hand, bi-Sobolev mappings do not enjoy such a property; it may happen that their Jacobian vanishes on a set of positive measure ([19], [20], [14]).

The bi-Sobolev assumption rules out the Lipschitz homeomorphism

$$
f_{0}:(0,2) \times(0,1) \rightarrow(0,1) \times(0,1), \quad f_{0}(x, y)=(h(x), y),
$$

where $h^{-1}(t)=t+c(t)$ and $c:(0,1) \rightarrow(0,1)$ is the usual Cantor ternary function because $f_{0}^{-1}$ does not belong to $\mathscr{W}_{\text {loc }}^{1,1}$. On the contrary, our first results deal with $\mathscr{W}^{1,1}$-homeomorphisms which include $f_{0}$ as well (Theorem 1.3). Another interesting property of a bi-Sobolev map $f=(u, v)$ in the plane is that $u$ and $v$ have the same critical points ([13], [17]).

Theorem 1.2. Let $f: \Omega \stackrel{\text { onto }}{\longrightarrow} \Omega^{\prime}$ be a bi-Sobolev map $f=(u, v)$. Then $u$ and $v$ have the same critical points:

$$
\{z \in \Omega:|\nabla u(z)|=0\}=\{z \in \Omega:|\nabla v(z)|=0\} \quad \text { a.e. }
$$

The same result holds also for the inverse $f^{-1}$. The analogue of this Theorem is not valid in more than two dimensions (see [13]).

Let us point out that we only assume that $f$ and $f^{-1}$ are in $\mathscr{W}_{\text {loc }}^{1,1}$. In the category of $\mathscr{W}^{1, p}$-bi-Sobolev maps, that is, $f$ belongs to $\mathscr{W}_{\text {loc }}^{1, p}\left(\Omega ; \mathbf{R}^{2}\right)$ and $f^{-1}$ belongs to $\mathscr{W}_{\text {loc }}^{1, p}\left(\Omega^{\prime} ; \mathbf{R}^{2}\right)$, the case $1 \leq p<2$ (see [20]) is critical with respect to the so-called $\mathrm{N}$ property of Lusin, i.e., that a function maps every set of measure zero to a set of measure zero. Let us mention that for $\mathscr{W}^{1,2}$-bi-Sobolev mappings the statement of Theorem 1.2 is obviously satisfied. In fact (see [16], p. 150), for homeomorphisms in $\mathscr{W}_{\text {loc }}^{1,2}$ we have the $\mathrm{N}$ property. Clearly

$$
\{z \in \Omega:|\nabla u(z)|=0\} \subset\left\{z \in \Omega: J_{f}(z)=0\right\} \quad \text { a.e. }
$$

We can decompose the set $\left\{J_{f}=0\right\}$ into a null set $Z$ and countably many sets on which we can use the Sard's Lemma (see [4], Theorem 3.1.8). It follows that

$$
\left|f\left(\left\{J_{f}=0\right\} \backslash Z\right)\right|=0 \text { and hence }|f(\{\nabla u=0\} \backslash Z)|=0 .
$$

Since $f^{-1}$ satisfies the $\mathrm{N}$ property, we obtain $|\{\nabla u=0\}|=0$ and analogously $|\{\nabla v=0\}|=0$ as well.

We observe that the following identity

$$
\left\{z \in \Omega:\left|\frac{\partial f}{\partial x}(z)\right|=0\right\}=\left\{z \in \Omega:\left|\frac{\partial f}{\partial y}(z)\right|=0\right\} \quad \text { a.e. }
$$

where $\left|\frac{\partial f}{\partial x}(z)\right|^{2}=u_{x}^{2}(z)+v_{x}^{2}(z)$ and $\left|\frac{\partial f}{\partial y}(z)\right|^{2}=u_{y}^{2}(z)+v_{y}^{2}(z)$, is true for bi-Sobolev maps and parallels (1.5). This is a consequence of the following characteristic property of a bi-Sobolev map which was proved in [3], [13], [9]:

$$
J_{f}(z)=0 \quad \Longrightarrow \quad|D f(z)|=0 \quad \text { a.e. }
$$

Our first result is the following, in which we give some identities for $\mathscr{W}^{1,1}$ homeomorphism. Notice that the symbol $|\nabla \varphi|\left(\Omega^{\prime}\right)$ denotes the total variation of 
the real function $\varphi$ belonging to the space $\mathrm{BV}\left(\Omega^{\prime}\right)$ of functions of bounded variation on $\Omega^{\prime}$ (see Section 2).

Theorem 1.3. Let $f=(u, v): \Omega \subset \mathbf{R}^{2} \stackrel{\text { onto }}{\longrightarrow} \Omega^{\prime} \subset \mathbf{R}^{2}$ be a homeomorphism whose inverse is $f^{-1}=(x, y)$. If we assume $u, v \in \mathscr{W}_{\text {loc }}^{1,1}(\Omega)$, then $x, y \in \operatorname{BV}_{\text {loc }}\left(\Omega^{\prime}\right)$ and

$$
\begin{aligned}
|\nabla y|\left(\Omega^{\prime}\right) & =\int_{\Omega}\left|\frac{\partial f}{\partial x}(z)\right| d z \\
|\nabla x|\left(\Omega^{\prime}\right) & =\int_{\Omega}\left|\frac{\partial f}{\partial y}(z)\right| d z .
\end{aligned}
$$

In [11] it was proved that if $f: \Omega \subset \mathbf{R}^{2} \stackrel{\text { onto }}{\longrightarrow} \Omega^{\prime} \subset \mathbf{R}^{2}$ has bounded variation, $f \in \mathrm{BV}_{\text {loc }}\left(\Omega ; \mathbf{R}^{2}\right)$, then $f^{-1} \in \mathrm{BV}_{\text {loc }}\left(\Omega^{\prime} ; \mathbf{R}^{2}\right)$ and both $f$ and $f^{-1}$ are differentiable a.e. We notice that our identities (1.7) and (1.8) represent an improvement of such a result when $f$ is $\mathscr{W}^{1,1}$-homeomorphism; in particular the following estimate

$$
\left|D f^{-1}\right|\left(\Omega^{\prime}\right) \leq 2 \int_{\Omega}|D f| d z
$$

holds (Corollary 3.4). A $\mathscr{W}_{\text {loc }}^{1, p}$-homeomorphism in the plane, $1 \leq p<2$ whose Jacobian vanishes a.e., has been recently constructed by Hencl [8]; such a mapping satisfies the assumptions of Theorem 1.3. If in Theorem 1.3 we add the hypothesis $J_{f}>0$ a.e., we obtain the identities (1.7) and (1.8) using the area formula (see Sections 2 and 3$)$.

Condition (1.6) makes it possible, for a given bi-Sobolev mapping $f$, to consider the distortion quotient

$$
\frac{|D f(z)|^{2}}{J_{f}(z)} \quad \text { for a.e. } z \in \Omega .
$$

Hereafter the undetermined ratio $\frac{0}{0}$ is understood to be equal to 1 for $z$ in the zero set of the Jacobian. The Borel function

$$
K_{f}(z):= \begin{cases}\frac{|D f(z)|^{2}}{J_{f}(z)} & \text { if } J_{f}(z)>0 \\ 1 & \text { otherwise, }\end{cases}
$$

is the distortion function of $f$ and has relevant properties: it is the smallest function $K(z)$ greater or equal to 1 for which the distortion inequality:

$$
|D f(z)|^{2} \leq K(z) J_{f}(z) \quad \text { a.e. } z \in \Omega
$$

holds true. Moreover, there are interesting interplay between the integrability of the distortions $K_{f}$ and $K_{f^{-1}}$ and the regularity of $f$ and $f^{-1}$ (see [13], Theorem 5).

In our general context of $\mathscr{W}^{1,1}$-homeomorphisms there are different distortion functions which play a significant role (see Section 4). We obtain conditions under which one of these functions is finite a.e. or integrable.

\section{Preliminaries}

We denote by $|A|$ the Lebesgue measure of a set $A \subset \mathbf{R}^{2}$. We say that two sets $A, B \subseteq \mathbf{R}^{2}$ satisfy $A=B$ a.e. if their symmetrical difference has measure zero, i.e.,

$$
|(A \backslash B) \cup(B \backslash A)|=0 .
$$


A homeomorphic mapping $f: \Omega \subset \mathbf{R}^{2} \stackrel{\text { onto }}{\longrightarrow} \Omega^{\prime} \subset \mathbf{R}^{2}$ is said to satisfy the $\mathrm{N}$ property of Lusin on the domain $\Omega$ if for every $A \subset \Omega$ such that $|A|=0$ we have $|f(A)|=0$.

A function $u \in \mathscr{L}^{1}(\Omega)$ is of bounded variation, $u \in \mathrm{BV}(\Omega)$ if the distributional partial derivatives of $u$ are measures with finite total variation in $\Omega$ : there exist Radon signed measures $D_{1} u, D_{2} u$ in $\Omega$ such that for $i=1,2,\left|D_{i} u\right|(\Omega)<\infty$ and

$$
\int_{\Omega} u D_{i} \phi(z) d z=-\int_{\Omega} \phi(z) d D_{i} u(z) \quad \forall \phi \in C_{0}^{1}(\Omega) .
$$

The gradient of $u$ is then a vector-valued measure with finite total variation

$$
|\nabla u|(\Omega)=\sup \left\{\int_{\Omega} u \operatorname{div} \varphi(z) d z: \varphi \in C_{0}^{1}\left(\Omega, \mathbf{R}^{2}\right),\|\varphi\|_{\infty} \leq 1\right\}<\infty .
$$

By $|\nabla u|$ we denote the total variation of the signed measure $D u$.

The Sobolev space $\mathscr{W}^{1,1}(\Omega)$ is contained in $\operatorname{BV}(\Omega)$; indeed for any $u \in \mathscr{W}^{1,1}(\Omega)$ the total variation is given by $\int_{\Omega}|\nabla u|=|\nabla u|(\Omega)$. We say that $f=(u, v) \in$ $\mathscr{L}^{1}\left(\Omega ; \mathbf{R}^{2}\right)$ belongs to $\mathrm{BV}\left(\Omega ; \mathbf{R}^{2}\right)$ if $u, v \in \mathrm{BV}(\Omega)$. Finally we say that $f \in \mathrm{BV}_{\text {loc }}\left(\Omega ; \mathbf{R}^{2}\right)$ if $f \in \mathrm{BV}\left(A ; \mathbf{R}^{2}\right)$ for every open $A \subset \subset \Omega$. In the following, for $f \in \mathrm{BV}_{\text {loc }}\left(\Omega ; \mathbf{R}^{2}\right)$ we will denote the total variation of $f$ by:

$$
|D f|(\Omega)=\sup \left\{\int_{\Omega} u \operatorname{div} \varphi_{1}(z) d z+\int_{\Omega} v \operatorname{div} \varphi_{2}(z) d z: \varphi_{i} \in C_{0}^{1}\left(\Omega ; \mathbf{R}^{2}\right),\left\|\varphi_{i}\right\|_{\infty} \leq 1, i=1,2\right\} .
$$

We will need the definition of sets of finite perimeter (see [1]).

Definition 2.1. Let $E$ be a Lebesgue measurable subset of $\mathbf{R}^{2}$. For any open set $\Omega \subset \mathbf{R}^{2}$ the perimeter of $E$ in $\Omega$, denoted by $P(E, \Omega)$, is the total variation of $\chi_{E}$ in $\Omega$, i.e.,

$$
P(E, \Omega)=\sup \left\{\int_{E} \operatorname{div} \varphi d z: \varphi \in C_{0}^{1}\left(\Omega ; \mathbf{R}^{2}\right),\|\varphi\|_{\infty} \leq 1\right\}
$$

We say that $E$ is a set of finite perimeter in $\Omega$ if $P(E, \Omega)<\infty$.

We say that $f=(u, v) \in \mathscr{W}_{\text {loc }}^{1, p}\left(\Omega ; \mathbf{R}^{2}\right), 1 \leq p \leq \infty$, if for each open $A \subset \subset \Omega, f$ belongs to the Sobolev space $\mathscr{W}^{1, p}\left(A ; \mathbf{R}^{2}\right)$, i.e., if $u \in \mathscr{L}^{p}(A)$ and $v \in \mathscr{L}^{p}(A)$ have distributional derivatives in $\mathscr{L}^{p}(A)$.

We are interested in the area formula for a homeomorphism $f \in \mathscr{W}_{\text {loc }}^{1,1}\left(\Omega ; \mathbf{R}^{2}\right)$ with $\Omega \subset \mathbf{R}^{2}$. In this case we have

$$
\int_{\Omega} \eta(f(z)) J_{f}(z) d z \leq \int_{\mathbf{R}^{2}} \eta(w) d w
$$

for any non negative Borel function $\eta$ on $\mathbf{R}^{2}$. This follows from the area formula for Lipschitz mappings (see [4], Theorem 3.2.3), and from a general property of a.e. differentiable functions (see [4], Theorem 3.1.8), namely that $\Omega$ can be exhausted up to a set of measure zero by sets the restriction to which of $f$ is Lipschitz continuous.

Moreover, the area formula

$$
\int_{E} \eta(f(z)) J_{f}(z) d z=\int_{\mathbf{R}^{2}} \eta(w) d w
$$

holds on each set $E \subset \Omega$ on which the N property of Lusin is satisfied. 


\section{The identities for $\mathscr{W}^{1,1}$-homeomorphisms}

Before proving Theorem 1.3 in its full generality we give now a partial proof under the following additional assumptions:

$$
\begin{aligned}
\left\{w: J_{f^{-1}}(w)\right. & =0\}=\{w:|\nabla y(w)|=0\} \quad \text { a.e. } \\
\left\{z: J_{f}(z)\right. & =0\}=\left\{z:\left|\frac{\partial f}{\partial x}(z)\right|=0\right\} \quad \text { a.e. }
\end{aligned}
$$

where $J_{f^{-1}}$ denotes the determinant of the absolutely continuous part of $D f^{-1}$; moreover, we suppose $f^{-1}$ differentiable a.e. in the classical sense. Therefore, we have

$$
\int_{\Omega^{\prime}}|\nabla y(w)| d w=\int_{A^{\prime}}|\nabla y(w)| d w
$$

where $A^{\prime}$ is a Borel subset of the set $E^{\prime}$ where $f^{-1}$ is differentiable with $J_{f^{-1}}>0$ such that $\left|A^{\prime}\right|=\left|E^{\prime}\right|$.

Applying (2.1), (3.1) and basic linear algebra, we arrive at:

$$
\begin{aligned}
\int_{A^{\prime}}|\nabla y(w)| d w & =\int_{A^{\prime}} \frac{|\nabla y(w)|}{J_{f^{-1}}(w)} J_{f^{-1}}(w) d w \leq \int_{f^{-1}\left(A^{\prime}\right)} \frac{|\nabla y(f(z))|}{J_{f^{-1}(f(z))}(z} \\
& =\int_{f^{-1}\left(A^{\prime}\right)}\left|\frac{\partial f}{\partial x}(z)\right| d z \leq \int_{\Omega}\left|\frac{\partial f}{\partial x}(z)\right| d z .
\end{aligned}
$$

Here we are using the identity $D \operatorname{adj} D=I \operatorname{det} D$ and the fact that $J_{f}(z) J_{f^{-1}}(f(z))=$ 1 at the points of differentiability with nonzero Jacobian. We have used as well the expression of the inverse matrix to the differential $2 \times 2$ matrix $D f=\left(\begin{array}{ll}u_{x} & u_{y} \\ v_{x} & v_{y}\end{array}\right)$ in terms of $D f^{-1}=\left(\begin{array}{ll}x_{u} & x_{v} \\ y_{u} & y_{v}\end{array}\right)$, namely

$$
y_{u}(f(z))=-v_{x}(z) J_{f^{-1}}(f(z)), \quad y_{v}(f(z))=u_{x}(z) J_{f^{-1}}(f(z)) \quad \forall z \in f^{-1}\left(A^{\prime}\right),
$$

and the identity

$$
|\nabla y(f(z))|^{2}=\left[v_{x}(z)^{2}+u_{x}(z)^{2}\right] J_{f^{-1}}(f(z))^{2} \quad \forall z \in f^{-1}\left(A^{\prime}\right) .
$$

The opposite inequality follows by a symmetric procedure which relies on (3.2).

Notice that (3.1) and (3.2) are certainly satisfied if $J_{f}>0$ a.e. and $J_{f^{-1}}>0$ a.e. We observe that Theorem 1.1 can be proved using the same technique under the additional assumptions that $J_{f}>0$ and $J_{f^{-1}}>0$ a.e. In the general case the proof of Theorem 1.3 is completely different; to prove the Theorem we need some preliminary results. The next Lemma is known as Coarea Formula (see [1], Theorem 3.40):

Lemma 3.1. For any open set $\Omega^{\prime} \subset \mathbf{R}^{2}$ and $y \in \mathscr{L}_{\text {loc }}^{1}\left(\Omega^{\prime}\right)$ we have

$$
|\nabla y|\left(\Omega^{\prime}\right)=\int_{-\infty}^{+\infty} P\left(\left\{w \in \Omega^{\prime}: y(w)>t\right\}, \Omega^{\prime}\right) d t .
$$

We understand the left-hand side of (3.3) to be infinity if $y \notin \mathrm{BV}$.

The following Lemma is the main step towards the equality in the area formula (see Theorem 1.3 of [3] and also [13], where the case $f$ ACL, i.e., absolutely continuous on lines, is treated). 
Lemma 3.2. Let $f \in \mathscr{W}_{\text {loc }}^{1,1}\left((-1,1)^{2} ; \mathbf{R}^{2}\right)$ be a homeomorphism. Then for almost every $t \in(-1,1)$ the mapping $f_{\mid(-1,1) \times\{t\}}$ satisfies the $N$ property of Lusin, i.e., for every $A \subset(-1,1) \times\{t\}, \mathscr{H}^{1}(A)=0$ implies $\mathscr{H}^{1}(f(A))=0$.

Proof of Theorem 1.3. Without loss of generality we take $\Omega=(-1,1) \times(-1,1)$. Let us apply Lemma 3.2 to the homeomorphism $f$. Then, the mapping

$$
f(\cdot, t): x \in(-1,1) \mapsto(u(x, t), v(x, t)) \in \Omega^{\prime}
$$

belongs to $\mathscr{W}^{1,1}\left((-1,1), \mathbf{R}^{2}\right)$ for a.e. $t$ and satisfies the $\mathrm{N}$ property. In particular, the area formula holds for $f(\cdot, t)$ on $(-1,1)$ :

$$
\int_{-1}^{1}\left|\frac{\partial f}{\partial x}(x, t)\right| d x=\mathscr{H}^{1}(f((-1,1) \times\{t\})) .
$$

Integrating with respect to $t$ we obtain:

$$
\int_{\Omega}\left|\frac{\partial f}{\partial x}(z)\right| d z=\int_{-1}^{1} \mathscr{H}^{1}(f((-1,1) \times\{t\})) d t .
$$

Since it is clear that

$$
f((-1,1) \times\{t\})=\left\{w \in \Omega^{\prime}: y(w)=t\right\}
$$

then

$$
\int_{\Omega}\left|\frac{\partial f}{\partial x}(z)\right| d z=\int_{-1}^{1} \mathscr{H}^{1}\left(\left\{w \in \Omega^{\prime}: y(w)=t\right\}\right) d t .
$$

As $y$ is continuous, then the set $\left\{w \in \Omega^{\prime}: y(w)=t\right\}$ is the boundary of the level set $\left\{w \in \Omega^{\prime}: y(w)>t\right\}$. By assumptions we know that for a.e. $t, \mathscr{H}^{1}\left(\left\{w \in \Omega^{\prime}: y(w)=\right.\right.$ t\}) $<\infty$ and from [1] (p. 209) we have

$$
\mathscr{H}^{1}\left(\left\{w \in \Omega^{\prime}: y(w)=t\right\}\right)=P\left(\left\{w \in \Omega^{\prime}: y(w)>t\right\}, \Omega^{\prime}\right) \quad \text { a.e. } t \in(-1,1) .
$$

Using Coarea Formula from Lemma 3.1, we obtain

$$
|\nabla y|\left(\Omega^{\prime}\right)=\int_{\Omega}\left|\frac{\partial f}{\partial x}(z)\right| d z
$$

and we deduce that $y \in \mathrm{BV}_{\text {loc }}\left(\Omega^{\prime}\right)$.

The equality (1.8) is proved using the same technique.

Remark 3.3. From the above proof it is clear that if $f$ is a homeomorphism in $\mathrm{BV}_{\text {loc }}\left(\Omega ; \mathbf{R}^{2}\right)$ such that $\frac{\partial f}{\partial x} \in \mathscr{L}^{1}\left(\Omega ; \mathbf{R}^{2}\right)$, then (1.7) holds true.

Since the total variation of a map is less or equal than the sum of total variation of the components, by Theorem 1.3 we immediately get

Corollary 3.4. Let $f=(u, v): \Omega \subset \mathbf{R}^{2} \stackrel{\text { onto }}{\longrightarrow} \Omega^{\prime} \subset \mathbf{R}^{2}$ be a homeomorphism whose inverse is $f^{-1}=(x, y)$. If we assume $u, v \in \mathscr{W}_{\text {loc }}^{1,1}(\Omega)$, then

$$
\left|D f^{-1}\right|\left(\Omega^{\prime}\right) \leq 2 \int_{\Omega}|D f|
$$




\section{The distortions of anisotropic Sobolev maps}

In Section 1 we have already mentioned the known fact that, if $f: \Omega \subset \mathbf{R}^{2} \stackrel{\text { onto }}{\longrightarrow}$ $\Omega^{\prime} \subset \mathbf{R}^{2}$ is bi-Sobolev, then we have

$$
\left\{z: J_{f}(z)=0\right\}=\{z:|D f(z)|=0\} \quad \text { a.e. }
$$

and this makes it possible to consider the distortion function

$$
K_{f}(z):= \begin{cases}\frac{|D f(z)|^{2}}{J_{f}(z)} & \text { if } J_{f}(z)>0 \\ 1 & \text { otherwise. }\end{cases}
$$

Moreover, the distortion inequality

$$
|D f(z)|^{2} \leq K_{f}(z) J_{f}(z)
$$

holds for a.e. $z \in \Omega$. According to a well established terminology, we say that $f$ has finite distortion $K_{f}$.

For a Sobolev homeomorphism, under suitable assumptions, it is possible to introduce different distortion functions (see [21]). Namely, if $f=(u, v)$ satisfies the condition

$$
\left\{z: J_{f}(z)=0\right\}=\{z:|\nabla u(z)|=0\} \quad \text { a.e., }
$$

then we are allowed to define the Borel function

$$
K_{f}^{(1)}(z):= \begin{cases}\frac{|\nabla u(z)|^{2}}{J_{f}(z)} & \text { if } J_{f}(z)>0 \\ 1 & \text { otherwise. }\end{cases}
$$

Similarly, if $f=(u, v)$ satisfies the condition

$$
\left\{z: J_{f}(z)=0\right\}=\{z:|\nabla v(z)|=0\} \quad \text { a.e. }
$$

then the Borel function

$$
K_{f}^{(2)}(z):= \begin{cases}\frac{|\nabla v(z)|^{2}}{J_{f}(z)} & \text { if } J_{f}(z)>0 \\ 1 & \text { otherwise }\end{cases}
$$

is well defined. On the other hand, if $f=(u, v)$ satisfies the condition

$$
\left\{z: J_{f}(z)=0\right\}=\left\{z:\left|\frac{\partial f}{\partial x}(z)\right|=0\right\} \quad \text { a.e. },
$$

then we can define the Borel function

$$
H_{f}^{(1)}(z):= \begin{cases}\frac{\left|\frac{\partial f}{\partial x}(z)\right|^{2}}{J_{f}(z)} & \text { if } J_{f}(z)>0 \\ 1 & \text { otherwise. }\end{cases}
$$

Finally, for $f$ satisfying

$$
\left\{z: J_{f}(z)=0\right\}=\left\{z:\left|\frac{\partial f}{\partial y}(z)\right|=0\right\} \quad \text { a.e. }
$$


we define

$$
H_{f}^{(2)}(z):= \begin{cases}\frac{\left|\frac{\partial f}{\partial y}(z)\right|^{2}}{J_{f}(z)} & \text { if } J_{f}(z)>0 \\ 1 & \text { otherwise. }\end{cases}
$$

In the following, given a $\mathscr{W}^{1,1}$-homeomorphism $f$, we establish conditions which guarantee that one of its distortions is finite a.e. or $\mathscr{L}^{1}$. Let us begin with the following

Theorem 4.1. Let $f=(u, v): \Omega \subset \mathbf{R}^{2} \stackrel{\text { onto }}{\longrightarrow} \Omega^{\prime} \subset \mathbf{R}^{2}$ be a $\mathscr{W}^{1,1}$ - homeomorphism whose inverse is $f^{-1}=(x, y)$. If $x \in \mathscr{W}_{\text {loc }}^{1,1}\left(\Omega^{\prime}\right)$ and $v_{y} \neq 0$ on a positive measure set $P \subset \Omega$, then

$$
\left\{z \in P: J_{f}(z)=0\right\}=\left\{z \in P:\left|\frac{\partial f}{\partial y}(z)\right|=0\right\} \quad \text { a.e. }
$$

and the distortion $H_{f}^{(2)}(z)$ is finite a.e. Moreover, we have the following identities

$$
\begin{aligned}
\int_{\Omega^{\prime}}|\nabla x(w)| d w & =\int_{\Omega}\left|\frac{\partial f}{\partial y}(z)\right| d z \\
|\nabla y(w)|\left(\Omega^{\prime}\right) & =\int_{\Omega}\left|\frac{\partial f}{\partial x}(z)\right| d z .
\end{aligned}
$$

Proof. By contradiction we suppose that there exists a set $A \subset P$ with positive Lebesgue measure such that $f$ is differentiable in $A$ and

$$
J_{f}(z)=0 \quad \text { and } \quad\left|\frac{\partial f}{\partial y}(z)\right|>0 \quad \forall z \in A .
$$

We can assume that $f$ is Lipschitz on $A$ and use the area formula (2.2) to get

$$
|f(A)|=0 \quad \text { since } \int_{A} J_{f}(z) d z=0 .
$$

We denote by

$$
p_{2}:\left(x_{1}, x_{2}\right) \in \mathbf{R}^{2} \rightarrow \mathbf{H}_{2}=\left\{x \in \mathbf{R}^{2}: x_{2}=0\right\}
$$

the orthogonal projection and by

$$
p^{(2)}:\left(x_{1}, x_{2}\right) \in \mathbf{R}^{2} \rightarrow x_{2} \in \mathbf{R}
$$

the second coordinate function.

We observe that

$$
\left\{\omega \in \Omega^{\prime}: x(\omega)=t\right\}=\left(p_{2} \circ f^{-1}\right)^{-1}\{(t, 0)\} \quad \forall t \in \mathbf{R} .
$$

By assumptions we know that

$$
\mathscr{H}^{1}(\{w \in f(A): x(w)=t\})<\infty
$$

and from [1] (p. 209)

$$
\mathscr{H}^{1}(\{w \in f(A): x(\omega)=t\})=P\left(\{w \in f(A): x(w)>t\}, \Omega^{\prime}\right) .
$$

By Lemma 3.1 and the assumption that $x$ belongs to $\mathscr{W}_{\text {loc }}^{1,1}\left(\Omega^{\prime}\right)$, we have

$$
\int_{\mathbf{R}} \mathscr{H}^{1}(\{w \in f(A): x(w)=t\})=\int_{f(A)}|\nabla x(\omega)| d w=0 .
$$


Thus the curve $\{w \in f(A): x(w)=t\}$ has zero one dimensional measure for a.e. $t \in \mathbf{R}$ and in particular its second projection to the axis have zero one-dimensional measure as well:

$$
\mathscr{H}^{1}\left(p^{(2)}(\{w \in f(A): x(\omega)=t\})\right)=0 \text { a.e. } t \in \mathbf{R} .
$$

On the other hand, using Fubini Theorem, we have

$$
|A|=\int_{\mathbf{R}}\left|A \cap p_{2}^{-1}\{(t, 0)\}\right| d t>0 .
$$

Hence, there exists $t_{0} \in \mathbf{R}$ such that

$$
\mathscr{H}^{1}\left(A \cap p_{2}^{-1}\left\{\left(t_{0}, 0\right)\right\}\right)>0 .
$$

Applying the area formula to the differentiable function $v\left(t_{0}, \cdot\right): \tau \in p^{(2)}(A) \rightarrow$ $v\left(t_{0}, \tau\right)$, we have

$$
\begin{aligned}
0 & <\int_{A \cap p_{2}^{-1}\left(t_{0}\right)}\left|v_{y}\left(t_{0}, \tau\right)\right| d \mathscr{H}^{1}(\tau) \leq \int_{\mathbf{R}} N\left(v, A \cap p_{2}^{-1}\left(t_{0}\right), \sigma\right) d \sigma \\
& =\int_{p^{(2)}\left(f(A) \cap\left(p_{2} \circ f^{-1}\right)^{-1}\left(t_{0}\right)\right)} N\left(v, A \cap p_{2}^{-1}\left(t_{0}\right), \sigma\right) d \sigma
\end{aligned}
$$

where $N\left(v, A \cap p_{2}^{-1}\left(t_{0}\right), \sigma\right)$ is the number of preimages of $\sigma$ under $v$ in $A \cap p_{2}^{-1}\left(t_{0}\right)$. The last integral is zero by (4.9) and this is a contradiction.

The following result shows that if the distortion $K_{f}^{(2)}$ is $\mathscr{L}^{1}$, then $f^{-1}$ has better Sobolev regularity.

Theorem 4.2. Let $f=(u, v): \Omega \subset \mathbf{R}^{2} \stackrel{\text { onto }}{\longrightarrow} \Omega^{\prime} \subset \mathbf{R}^{2}$ be a $\mathscr{W}^{1,1}$-homeomorphism and denote by $f^{-1}=(x, y)$ its inverse. If we assume

$$
\begin{gathered}
\left\{w \in \Omega^{\prime}: J_{f^{-1}}(w)=0\right\}=\left\{w \in \Omega^{\prime}:\left|\frac{\partial f^{-1}}{\partial u}(w)\right|=0\right\}, \\
\left\{z \in \Omega: J_{f}(z)=0\right\}=\{z \in \Omega:|\nabla v(z)|=0\}
\end{gathered}
$$

and $K_{f}^{(2)} \in \mathscr{L}^{1}$, then

$$
\left|\frac{\partial f^{-1}}{\partial u}\right| \in \mathscr{L}^{2}(\Omega)
$$

and

$$
\int_{\Omega^{\prime}}\left|\frac{\partial f^{-1}}{\partial u}(w)\right|^{2} d w \leq \int_{\Omega} K_{f}^{(2)}(z) d z .
$$

Proof. Let $A^{\prime}$ be the Borel subset of the set $E^{\prime}$ where $f^{-1}$ is differentiable with $J_{f^{-1}}>0$, such that $\left|A^{\prime}\right|=\left|E^{\prime}\right|$. Applying the area formula, we obtain

$$
\begin{aligned}
\int_{\Omega^{\prime}}\left|\frac{\partial f^{-1}}{\partial u}(w)\right|^{2} d w & =\int_{A^{\prime}}\left|\frac{\partial f^{-1}}{\partial u}(w)\right|^{2} d w=\int_{A^{\prime}} \frac{\left|\frac{\partial f^{-1}}{\partial u}(w)\right|^{2}}{J_{f^{-1}}(w)} J_{f^{-1}}(w) d w \\
& \leq \int_{f^{-1}\left(A^{\prime}\right)} \frac{\left|\frac{\partial f^{-1}}{\partial u}(f(z))\right|^{2}}{J_{f^{-1}}(f(z))} d z=\int_{f^{-1}\left(A^{\prime}\right)} \frac{\frac{1}{J_{f}(z)^{2}}|\nabla v(z)|^{2}}{J_{f^{-1}}(f(z))} d z
\end{aligned}
$$




$$
=\int_{f^{-1}\left(A^{\prime}\right)} \frac{|\nabla v(z)|^{2}}{J_{f}(z)} d z \leq \int_{\Omega^{\prime}} K_{f}^{(2)}(z) d z .
$$

\section{References}

[1] Ambrosio, L., N. Fusco, and D. Pallara: Functions of bounded variation and free discontinuity problems. - Oxford Math. Monogr., Oxford Univ. Press, New York, 2000.

[2] Astala, K., T. Iwaniec, and G. Martin: Elliptic partial differential equations and quasiconformal mappings in the plane. - Princeton Math. Ser. 48, Princeton Univ. Press, Princeton, NJ, 2009.

[3] Csörnyei, M., S. Hencl, and J. MalÝ: Homeomorphisms in the Sobolev space $W^{1, n-1}$. J. Reine Angew. Math. 644, 2010, 221-235.

[4] Federer, H.: Geometric measure theory. - Grundlehren Math. Wiss. 153, Springer-Verlag, New York, 1969 (second edition 1996).

[5] Fusco, N., G. Moscariello, and C. Sbordone: The limit of $\mathscr{W}^{1,1}$ homeomorphisms with finite distortion. - Calc. Var. Partial Differential Equations 33:3, 2008, 377-390.

[6] Gehring, F.W., and O. Lehto: On the total differentiability of functions of a complex variable. - Ann. Acad. Sci. Fenn. Ser. A I Math. 272, 1959, 1-9.

[7] Greco, L., C. Sbordone, and R. Schiattarella: Composition of bi-Sobolev homeomorphisms. - Proc. Roy. Soc. Edinburgh Sect. A (to appear).

[8] Hencl, S.: Sobolev homeomorphism with zero jacobian almost everywhere. - J. Math. Pures Appl. (to appear).

[9] Hencl, S., and P. Koskela: Regularity of the inverse of a planar Sobolev homeomorphism. - Arch. Ration. Mech. Anal. 180, 2006, 75-95.

[10] Hencl, S., P. Koskela, and J. Malý: Regularity of the inverse of a Sobolev homeomorphism in space. - Proc. Roy. Soc. Edinburgh Sect. A 136:6, 2006, 1267-1285.

[11] Hencl, S., P. Koskela, and J. Onninen: Homeomorphisms of bounded variation. - Arch. Ration. Mech. Anal. 186, 2007, 351-360.

[12] Hencl, S., and J. MALÝ: Jacobians of Sobolev homeomorphisms. - Calc. Var. Partial Differential Equations 38: 1-2, 2010, 233-242.

[13] Hencl, S., G. Moscariello, A. Passarelli di Napoli, and C. Sbordone: Bi-Sobolev mappings and elliptic equations in the plane. - J. Math. Anal. Appl. 355, 2009, 22-32.

[14] Iwaniec, T., and G. Martin: Geometric function theory and non-linear analysis. - Oxford Math. Monogr., The Clarendon Press, Oxford Univ. Press, New York, 2001.

[15] Koskela, P.: Regularity of the inverse of a Sobolev homeomorphism. - In: Proc. Internat. Congress of Mathematicians, Hyderabad, India, 2010.

[16] Lehto, O., and K. Virtanen: Quasiconformal mappings in the plane. - Springer-Verlag, Berlin, 1971.

[17] Moscariello, G., A. Passarelli di Napoli, and C. Sbordone: ACL-homeomorphisms in the plane. - Oper. Theory Adv. Appl. 193, 2009, 215-225.

[18] Moscariello, G., A. Passarelli di Napoli, and C. Sbordone: Planar ACL-homeomorphisms: critical points of their components. - Commun. Pure Appl. Anal. 9:5, 2010, 1391-1397.

[19] Ponomarev, S.: An example of an $A C T L^{p}$ homeomorphisms that is not absolutely continuous in the sense of Banach. - Dokl. Akad. Nauk SSSR 201, 1971, 1053-1054.

[20] Ponomarev, S.: On the N-property of homeomorphisms of the class $W_{p}^{1}$. - Sibirsk. Mat. Zh. 28, 1987, 140-148.

[21] Sbordone, C., and R. Schiattarella: Critical points for Sobolev homeomorphisms. - Rend. Mat. Accad. Lincei, 2011 (to appear).

Received 17 December 2010 